\title{
A criterion for convergence of solutions of homogeneous delay linear differential equations
}

\author{
by Josef Diblík (Brno)
}

Abstract. The linear homogeneous differential equation with variable delays

$$
\dot{y}(t)=\sum_{j=1}^{n} \alpha_{j}(t)\left[y(t)-y\left(t-\tau_{j}(t)\right)\right]
$$

is considered, where $\alpha_{j} \in C\left(I, \overline{\mathbb{R}^{+}}\right), I=\left[t_{0}, \infty\right), \mathbb{R}^{+}=(0, \infty), \sum_{j=1}^{n} \alpha_{j}(t)>0$ on $I$, $\tau_{j} \in C\left(I, \mathbb{R}^{+}\right)$, the functions $t-\tau_{j}(t), j=1, \ldots, n$, are increasing and the delays $\tau_{j}$ are bounded. A criterion and some sufficient conditions for convergence of all solutions of this equation are proved. The related problem of nonconvergence is also discussed. Some comparisons to known results are given.

1. Introduction and the main results. We shall deal with the linear homogeneous differential equation with variable delays

$$
\dot{y}(t)=\sum_{j=1}^{n} \alpha_{j}(t)\left[y(t)-y\left(t-\tau_{j}(t)\right)\right],
$$

where $\alpha_{j} \in C\left(I, \overline{\mathbb{R}^{+}}\right), I=\left[t_{0}, \infty\right), \mathbb{R}^{+}=(0, \infty), \sum_{j=1}^{n} \alpha_{j}(t)>0$ on $I$, $\tau_{j} \in C\left(I, \mathbb{R}^{+}\right)$, the functions $t-\tau_{j}(t), j=1, \ldots, n$, are increasing on $I$ and the delays $\tau_{j}$ are bounded on $I$, i.e. $\tau_{j}(t) \leq r=$ const, $t \in I$. Set $\tau(t)=$ $\max _{j}\left\{\tau_{j}(t)\right\}, t \in I, I_{p}=\left[t_{p}, \infty\right), t_{p+1}=t_{p}+\tau\left(t_{p+1}\right), p \in\{-1,0,1, \ldots\}$ and $I_{0} \equiv I$.

A function $y$ is called a solution of (1.1) corresponding to an initial point $t^{*} \in I$ if $y$ is defined and continuous on $\left[t^{*}-\tau\left(t^{*}\right), \infty\right)$, differentiable on $\left[t^{*}, \infty\right)$ and satisfies (1.1) for $t \geq t^{*}$. By a solution of (1.1) we mean a solution corresponding to some initial point $t^{*} \in I$. We denote by $y\left(t^{*}, \varphi\right)(t)$ a solution of (1.1) corresponding to the initial point $t^{*} \in I$ which is generated by a continuous initial function $\varphi:\left[t^{*}-\tau\left(t^{*}\right), t^{*}\right] \rightarrow \mathbb{R}$. In the

1991 Mathematics Subject Classification: 34K15, 34K25.

Key words and phrases: asymptotic convergence of solutions, linear homogeneous delay differential equation, topological principle of Ważewski (Rybakowski's approach). 
linear case the solution $y\left(t^{*}, \varphi\right)(t)$ is unique on its maximal existence interval $D_{t^{*}, \varphi}=\left[t^{*}, \infty\right)([10])$. We say that a solution of (1.1) corresponding to the initial point $t^{*}$ is convergent or asymptotically convergent if it has a finite limit at $\infty$.

Our aim in this paper is to formulate a criterion and some sufficient conditions for convergence of all solutions of (1.1). We also consider the related problem of nonconvergence of solutions of (1.1).

Problems concerning asymptotic constancy of solutions, asymptotic convergence of solutions or existence of asymptotic equilibrium of various classes of retarded functional differential equations were investigated e.g. by $\mathrm{O}$. Arino, I. Győri and M. Pituk [1], F. V. Atkinson and J. R. Haddock [2], R. Bellman and K. L. Cooke [3], I. Győri and M. Pituk [8, 9] and T. Krisztin [11]-[13]. Nonconvergence was considered e.g. by S. N. Zhang [18] and J. Diblík [7]. Some closely connected questions are discussed in the recent papers by J. Čermák $[5,6]$ (where, in some proofs, the fundamental results of F. Neuman $[14,15]$ concerning the transformation theory are used). In Section 2 some comparisons are given. Section 3 contains auxiliary lemmas and in the last Section 4 the proofs of the theorems are collected.

In this paper the following is proved:

Theorem 1 (Main Result). For the convergence of all solutions of (1.1) corresponding to an initial point $t_{0}$, a necessary and sufficient condition is that there exist functions $k_{i} \in C\left(I, \mathbb{R}^{+}\right), i=1, \ldots, n$, satisfying the system of integral inequalities

$$
1+k_{i}(t) \geq \exp \left[\int_{t-\tau_{i}(t)}^{t} \sum_{j=1}^{n} \alpha_{j}(s) k_{j}(s) d s\right], \quad i=1, \ldots, n,
$$

on the interval $I_{1}$.

The necessity part of this theorem can be formulated more precisely:

THEOREM 2. If all solutions of (1.1) corresponding to an initial point $t_{0}$ are convergent, then there are functions $k_{i} \in C\left(I, \mathbb{R}^{+}\right), i=1, \ldots, n$, satisfying $k_{i}(\infty)=0$ and the system of integral equations

$$
1+k_{i}(t)=\exp \left[\int_{t-\tau_{i}(t)}^{t} \sum_{j=1}^{n} \alpha_{j}(s) k_{j}(s) d s\right], \quad i=1, \ldots, n,
$$

on the interval $I_{1}$.

The following theorem establishes the fact that for convergence of all solutions of (1.1) the existence of only one nonconstant monotone convergent solution is sufficient. 
THEOREM 3. If there exists a nonconstant monotone convergent solution of (1.1) corresponding to an initial point $t_{0}$, then all solutions of (1.1) which correspond to $t_{0}$ are convergent.

Moreover, we prove the following result concerning nonconvergence of solutions of (1.1):

THEOREM 4 (Nonconvergence result). A sufficient and necessary condition for the existence of a solution of (1.1) corresponding to an initial point $t_{0}$ with the property $y(\infty)=\infty$ is the nonexistence of functions $k_{i} \in$ $C\left(I, \mathbb{R}^{+}\right), i=1, \ldots, n$, satisfying the system of integral inequalities (1.2) on the interval $I_{1}$.

The following theorem concerns (1.1) with bounded coefficients.

TheOREM 5. For convergence of all solutions of (1.1) corresponding to an initial point $t_{0}$, where $0<b \leq \alpha_{i}(t) \leq a, 0<\widetilde{r} \leq \tau_{i}(t), t \in I, a, b, \widetilde{r}=$ const, $i=1, \ldots, n$, the condition

$$
n b \widetilde{r}<1
$$

is necessary and the condition

$$
\text { nar }<1
$$

is sufficient.

2. Some consequences, comparisons and remarks. In this section we give some sufficient conditions for convergence and divergence of solutions of (1.1). These conditions are formulated in terms of the coefficients of (1.1) and their proofs are based on the results of the previous section. A comparison of these sufficient conditions with some known ones is given in Remark 1.

THEOREM 6. All solutions of (1.1) are convergent if

$$
\limsup _{t \rightarrow \infty} \int_{t-\tau_{i}(t)}^{t} \alpha_{j}(s) d s=q_{j i}
$$

where $i, j=1, \ldots, n, q_{j i}=$ const and $\sum_{j=1}^{n} q_{j i}<1, i=1, \ldots, n$.

THEOREM 7. For the existence of a solution of (1.1) corresponding to an initial point $t_{0}$ with the property $y(\infty)=\infty$, it is sufficient that

$$
\int_{t-\tau_{i}(t)}^{t} \alpha_{j}(s) d s \geq q_{j i}
$$

where $i, j=1, \ldots, n, t \in I_{1}, q_{j i}=$ const and $\sum_{j=1}^{n} q_{j i} \geq 1, i=1, \ldots, n$.

The next three theorems give more special convergence criteria. 
Theorem 8. Suppose $\tau_{j}(t) \leq \tau_{j}=$ const and

$$
\alpha_{j}(t) \leq \beta_{j}\left(\frac{1}{\tau_{j}}-\frac{L_{j}}{t}\right)
$$

for sufficiently large $t$, where $0<\beta_{j}, L_{j}=$ const, $j=1, \ldots, n, \sum_{j=1}^{n} \beta_{j}=1$ and $L_{j}>1 / 2$. Then each solution of (1.1) converges.

Example 1. Consider the equation

$$
\begin{aligned}
\dot{y}(t)= & \frac{1}{2}\left(1-\frac{L_{1}^{*}}{t}\right)[y(t)-y(t-1)] \\
& +\frac{1}{2}\left(\frac{1}{2}-\frac{L_{2}^{*}}{t}\right)[y(t)-y(t-2)],
\end{aligned}
$$

where $L_{1}^{*}, L_{2}^{*}$ are constants greater than $1 / 2$. Here $n=2, \tau_{1}=1, \tau_{2}=2$, $\alpha_{1}(t)=\left(1-L_{1}^{*} / t\right) / 2$ and $\alpha_{2}(t)=\left(0,5-L_{2}^{*} / t\right) / 2$. The assumption of Theorem 8 holds for $\beta_{1}=\beta_{2}=1 / 2$. Hence each solution of (2.3) converges.

Theorem 9. Suppose $\tau_{j}(t) \leq \tau=$ const, $j=1, \ldots, n$. If , moreover, for all sufficiently large $t$,

$$
\int_{t}^{t+\tau}\left(\sum_{j=1}^{n} \alpha_{j}(s)\right) d s \leq 1-\frac{\tau}{t}-\frac{\tau}{t \ln t}-\frac{L}{t \ln t \ln _{2} t}
$$

where $L>\tau, L=$ const and $\ln _{2} t=\ln \ln t$, then each solution of (1.1) converges.

Theorem 10. Suppose $\tau_{j}(t) \leq \tau=$ const, $j=1, \ldots, n$. If , moreover, for sufficiently large $t$,

$$
\int_{t}^{t+\tau}\left(\sum_{j=1}^{n} \alpha_{j}(s)\right) \frac{1}{s^{m}} d s \leq \frac{1}{t^{m}}-\frac{L}{t^{m+1}}
$$

where $m, L=$ const, $m \geq 1$ and $L>\tau m$, then each solution of (1.1) converges.

EXAMPLE 2. Consider the equation

$$
\dot{y}=\left[\frac{1}{2 \pi}\left(1+\frac{\sin t}{2}\right)-\frac{E}{t}\right] \cdot[y(t)-y(t-2 \pi)]
$$

where $E$ is a constant within the interval

$$
1>E>\frac{1}{2}\left(1+\frac{1}{2 \pi}\right) \doteq 0,5796 \text {. }
$$

Here $n=1, \tau_{1}=2 \pi$ and $\alpha_{1}(t)=(1+(\sin t) / 2) /(2 \pi)-E / t$. Let us show that neither the conditions of Theorem 8 nor those of Theorem 9 are valid but the assumptions of Theorem 10 are satisfied. 
Indeed, concerning Theorem 8 , we see that for sufficiently large $t$,

$$
\alpha_{1}(t)=\frac{1}{2 \pi}\left(1+\frac{\sin t}{2}\right)-\frac{E}{t} \leq \frac{1}{2 \pi} \cdot \frac{3}{2}-\frac{E}{t} \nless \frac{1}{2 \pi}-\frac{L_{1}}{t}
$$

with $L_{1}>1 / 2$, i.e. the inequality (2.2) does not hold.

Let us verify that the inequality (2.4) in Theorem 9 , with $\tau=2 \pi$, does not hold either: indeed, for $t \rightarrow \infty$ and the indicated values of $E$,

$$
\begin{aligned}
\int_{t}^{t+\tau} \alpha_{1}(s) d s & =\int_{t}^{t+2 \pi}\left(\frac{1}{2 \pi}\left(1+\frac{\sin s}{2}\right)-\frac{E}{s}\right) d s=1-E \ln \left(1+\frac{2 \pi}{t}\right) \\
& =1-2 \pi E \cdot \frac{1}{t}+O\left(\frac{1}{t^{2}}\right) \nless 1-\frac{2 \pi}{t}-\frac{2 \pi}{t \ln t}-\frac{L}{t \ln t \ln _{2} t}
\end{aligned}
$$

with $L>2 \pi$.

Now let us verify the inequality (2.5) with $m=1$ and $\tau=2 \pi$. An easy computation gives

$$
\begin{aligned}
\int_{t}^{t+\tau} \alpha_{1}(s) \frac{1}{s} d s= & \int_{t}^{t+2 \pi}\left(\frac{1}{2 \pi}\left(1+\frac{\sin s}{2}\right)-\frac{E}{s}\right) \cdot \frac{1}{s} d s \\
= & \frac{1}{2 \pi} \ln \left(1+\frac{2 \pi}{t}\right)+\frac{1}{4 \pi} \int_{t}^{t+2 \pi} \frac{\sin s}{s} d s-E \int_{t}^{t+2 \pi} \frac{1}{s^{2}} d s \\
= & \frac{1}{2 \pi}\left(\frac{2 \pi}{t}-\frac{(2 \pi)^{2}}{2 t^{2}}+O\left(\frac{1}{t^{3}}\right)\right) \\
& +\frac{1}{4 \pi}\left[-\left.\frac{\cos s}{s}\right|_{t} ^{t+2 \pi}-\left.\frac{\sin s}{s^{2}}\right|_{t} ^{t+2 \pi}-2 \int_{t}^{t+2 \pi} \frac{\sin s}{s^{3}} d s\right]+\left.\frac{E}{s}\right|_{t} ^{t+2 \pi} \\
= & \frac{1}{t}-\frac{\pi(1+2 E)}{t^{2}}+\frac{\cos t}{2 t^{2}}+O\left(\frac{1}{t^{3}}\right) \\
\leq & \frac{1}{t}+\left(-\pi(1+2 E)+\frac{1}{2}\right) \frac{1}{t^{2}}+O\left(\frac{1}{t^{3}}\right)
\end{aligned}
$$

For the validity of $(2.5)$ it is now sufficient that

$$
-\pi(1+2 E)+\frac{1}{2}<-2 \pi
$$

i.e. $E>\frac{1}{2}\left(1+\frac{1}{2 \pi}\right)$, and so, by Theorem 10, all solutions of (2.6) converge.

Example 3. Consider the equation

$$
\dot{y}=\left(\frac{1}{\tau}-\frac{a}{t}\right) \cdot[y(t)-y(t-\tau)]
$$

where $0<\tau=$ const and $a>0$ is a constant. Let us find the values of $a$ for which we have convergence of all solutions of (2.7). Here $n=1$ and $\tau_{1}=\tau$. 
Theorem 8 and also Theorem 10 (with $m=1$ ) guarantee the convergence for $a>1 / 2$. Theorem 9 gives only $a>1$. Note that the value $a=1 / 2$ is the best possible. Indeed (see [7], Remark 1), for $a \in(0,1 / 2]$ there exists a solution $y=Y(t)$ of $(2.7)$ with $Y(\infty)=\infty$.

REMARK 1 . Theorems 6,8 and 9 generalize some special criteria given by F. V. Atkinson and J. R. Haddock [2]. Theorem 7 gives a generalization of a result of S. N. Zhang [18]. Theorem 10 gives a new (and, as shown by Examples 2 and 3, very efficient) result in this direction.

REMARK 2. Note that each convergent solution $y=y(t)$ of (1.1) (existence of which was established by Theorems 1, 3, 6 and 8-10) satisfies

$$
|y(t)-y(\infty)|<\psi(t), \quad t \in I,
$$

where the positive function $\psi$ with $\psi(\infty)=0$ is defined in the sufficiency part of the proof of Theorem 1 .

REMARK 3. The importance of the investigation of (1.1) consists, among other things, in the fact that it is connected with the investigation of the equation

$$
\dot{x}(t)=-\sum_{j=1}^{n} \beta_{j}(t) x\left(t-\tau_{j}(t)\right),
$$

where $\beta_{j} \in C\left(I, \overline{\mathbb{R}^{+}}\right), j=1, \ldots, n$, and $\sum_{j=1}^{n} \beta_{j}(t)>0$ on $I$. Indeed, if there exists a positive solution $x=\omega(t)$ of the latter equation, then the transformation $x=\omega(t) y$ gives an equation of type (1.1) where $\alpha_{j}(t) \equiv$ $\beta_{j}(t) \omega\left(t-\tau_{j}(t)\right) \omega^{-1}(t), j=1, \ldots, n$, i.e.

$$
\dot{y}(t)=\frac{1}{\omega(t)} \sum_{j=1}^{n} \beta_{j}(t) \omega\left(t-\tau_{j}(t)\right)\left[y(t)-y\left(t-\tau_{j}(t)\right)\right] .
$$

3. Lemmas. We start with the following lemmas. Obviously in view of the form of (1.1) we have

LEMma 1. If $y\left(t^{*}, \varphi\right)(t)$ is a solution of (1.1) corresponding to an initial point $t^{*} \in I$, then the function $y\left(t^{*}, \psi\right)(t)$, where $\psi(t)=K \varphi(t)+L$, is a solution of (1.1) corresponding to the initial point $t^{*}$ for any constants $K, L \in \mathbb{R}$.

Lemma 2. Let the initial function $\varphi(t)$ be defined and continuous on the interval $\left[t_{p}, t_{p+1}\right]$ for some $p \in\{-1,0,1, \ldots\}$ and either

$$
\varphi(t)<\varphi\left(t_{p+1}\right)
$$

or

$$
\varphi(t)>\varphi\left(t_{p+1}\right)
$$


for all $t \in\left[t_{p}, t_{p+1}\right)$. Then the corresponding solution $y\left(t_{p+1}, \varphi\right)(t)$ is increasing on $I_{p+1}$ in the case (3.1), and decreasing on $I_{p+1}$ in the case (3.2).

P r o of. It follows from (1.1) that sign $\dot{y}\left(t_{p+1}, \varphi\right)\left(t_{p+1}+0\right)=+1$ in the case (3.1) and sign $\dot{y}\left(t_{p+1}, \varphi\right)\left(t_{p+1}+0\right)=-1$ in the case (3.2). The case $\dot{y}\left(t_{p+1}, \varphi\right)\left(t^{\star}\right)=0$ for a $t^{\star} \in\left(t_{p+1}, \infty\right)$ and $\operatorname{sign} \dot{y}\left(t_{p+1}, \varphi\right)(t) \neq 0$ for all $t \in\left(t_{p+1}, t^{\star}\right)$ is impossible because, by (1.1), we have $y\left(t_{p+1}, \varphi\right)\left(t^{\star}\right) \neq$ $y\left(t_{p+1}, \varphi\right)\left(t^{\star}-\tau\left(t^{\star}\right)\right)$.

Consider the system of functional differential equations with retarded argument

$$
\dot{y}(t)=f\left(t, y_{t}\right)
$$

where $y(t) \in \mathbb{R}^{n}, y_{t} \in C=C\left([-r, 0], \mathbb{R}^{n}\right), y_{t}(\theta)=y(t+\theta)$ for $\theta \in[-r, 0]$, $f: \Omega \rightarrow \mathbb{R}^{n}, \Omega$ is an open subset of $\mathbb{R} \times C$ and $f$ is a continuous mapping such that the element $(\delta, \pi) \in \Omega$ determines a unique solution $y(\delta, \pi)$ on its maximal existence interval $D_{\delta, \pi}=[\delta, a), \delta<a \leq \infty$ (see e.g. [10]).

In the proof of the sufficiency part of Theorem 1 the topological principle of T. Ważewski [17] in the form of K. P. Rybakowski [16] (for retarded functional differential equations) is used. A summary of this principle is given below. (As usual, if $\omega \subset \mathbb{R} \times \mathbb{R}^{n}$, then int $\omega$ and $\partial \omega$ denote the interior and the boundary of $\omega$, respectively.)

Definition 1 [16]. Let $l_{i}(t, y), i=1, \ldots, p$, and $m_{j}(t, y), j=1, \ldots, q$, be continuously differentiable functions defined on some open set $\omega_{0} \subset \mathbb{R} \times \mathbb{R}^{n}$. The set

$$
\omega=\left\{(t, y) \in \omega_{0}: l_{i}(t, y)<0, m_{j}(t, y)<0, i=1, \ldots, p, j=1, \ldots, q\right\}
$$

is called a regular polyfacial set with respect to the system (3.3) if $(\alpha)$ to $(\gamma)$ below hold:

$(\alpha)$ For $(t, \pi) \in \mathbb{R} \times C$ such that $(t+\theta, \pi(\theta)) \in \omega$ for all $\theta \in[-r, 0)$, we have $(t, \pi) \in \Omega$.

$(\beta)$ For all $i=1, \ldots, p$, all $(t, y) \in \partial \omega$ for which $l_{i}(t, y)=0$, and all $\pi \in C$ for which $\pi(0)=y$ and $(t+\theta, \pi(\theta)) \in \omega$ for all $\theta \in[-r, 0)$, we have

$$
D l_{i}(t, y) \equiv \sum_{r=1}^{n} \frac{\partial l_{i}(t, y)}{\partial y_{r}} f_{r}(t, \pi)+\frac{\partial l_{i}(t, y)}{\partial t}>0 .
$$

$(\gamma)$ For all $j=1, \ldots, q$, all $(t, y) \in \partial \omega$ for which $m_{j}(t, y)=0$, and all $\pi \in C$ for which $\pi(0)=y$ and $(t+\theta, \pi(\theta)) \in \omega$ for all $\theta \in[-r, 0)$, we have

$$
D m_{j}(t, y) \equiv \sum_{r=1}^{n} \frac{\partial m_{j}(t, y)}{\partial y_{r}} f_{r}(t, \pi)+\frac{\partial m_{j}(t, y)}{\partial t}<0 \text {. }
$$


In the sequel the elements $(t, \pi) \in \mathbb{R} \times C$ are assumed to be such that $(t, \pi) \in \Omega$.

Definition 2. A system of initial functions $p_{A, \omega}$ with respect to nonempty sets $A$ and $\omega$ where $A \subset \bar{\omega} \subset \mathbb{R} \times \mathbb{R}^{n}$ is defined to be a continuous mapping $p: A \rightarrow C$ such that $(\alpha)$ and $(\beta)$ below hold:

$(\alpha)$ If $z=(t, y) \in A \cap \operatorname{int} \omega$, then $(t+\theta, p(z)(\theta)) \in \omega$ for all $\theta \in[-r, 0]$.

$(\beta)$ If $z=(t, y) \in A \cap \partial \omega$, then $(t+\theta, p(z)(\theta)) \in \omega$ for all $\theta \in[-r, 0)$ and $(t, p(z)(0))=z$.

The following lemma is the main result of [16].

Lemma 3. Let $\omega \subset \omega_{0}$ be a regular polyfacial set with respect to the system (3.3) and set

$$
W=\left\{(t, y) \in \partial \omega: m_{j}(t, y)<0, j=1, \ldots, q\right\} .
$$

Let $Z \subset W \cup \omega$ be such that $Z \cap W$ is a retract of $W$ but not a retract of $Z$. If a system of initial functions $p_{Z, \omega}$ is fixed, then there exists $z_{0}=\left(\sigma_{0}, y_{0}\right) \in$ $Z \cap \omega$ such that for the corresponding solution $y\left(\sigma_{0}, p\left(z_{0}\right)\right)(t)$ of $(3.3)$ we have $\left(t, y\left(\sigma_{0}, p\left(z_{0}\right)\right)(t)\right) \in \omega$ for each $t \in D_{\sigma_{0}, p\left(z_{0}\right)}$.

\section{Proofs of the theorems}

Proof of Theorem 1. Necessity. Suppose all solutions are convergent. By Lemmas 1, 2 there is a convergent solution $y=y(t)$ corresponding to the initial point $t_{0}$ which is generated by a decreasing initial function and, moreover, this solution is decreasing on $I_{-1}$ with positive limit. Integrate (1.1) (after dividing by $y(t)$ ) with limits $t$ and $t-\tau_{i}(t)$ to obtain

$$
\frac{y\left(t-\tau_{i}(t)\right)}{y(t)}=\exp \left\{\int_{t-\tau_{i}(t)}^{t} \sum_{j=1}^{n} \alpha_{j}(s)\left[-1+\frac{y\left(s-\tau_{j}(s)\right)}{y(s)}\right] d s\right\}
$$

for $t \in I_{1}, i=1, \ldots, n$.

Define

$$
k_{i}(t) \equiv-1+\frac{y\left(t-\tau_{i}(t)\right)}{y(t)}, \quad i=1, \ldots, n, t \in I .
$$

This ends the proof of necessity since $y(t)$ is decreasing and so $k_{i}(t)>0$, $i=1, \ldots, n$, on $I$.

Sufficiency. Let us exclude the trivial case when a convergent solution is generated by a constant initial function, i.e. let us exclude the set of all constant solutions. In the sequel, we consider a solution $y\left(t_{0}, \varphi\right)(t)$ of (1.1) which corresponds to a nonconstant initial continuous function $\varphi(t)$, $t \in\left[t_{-1}, t_{0}\right]$. Suppose that there are functions $k_{i} \in C\left(I, \mathbb{R}^{+}\right), i=1, \ldots, n$, which satisfy (1.2). Define the auxiliary function 


$$
\psi(t)=\left\{\begin{array}{rr}
\delta \exp \left[-\int_{t_{0}}^{t} \sum_{j=1}^{n} \alpha_{j}(s) k_{j}(s) d s\right] & \text { if } \int_{j=1}^{\infty} \sum_{j}^{n} \alpha_{j}(t) k_{j}(t) d t=\infty, \\
\delta \exp \left[-\int_{t_{0}}^{t} \sum_{j=1}^{n} \alpha_{j}(s) k_{j}(s) d s\right]-\delta \exp \left[-\int_{t_{0}}^{\infty} \sum_{j=1}^{n} \alpha_{j}(s) k_{j}(s) d s\right] \\
& \text { if } \int^{\infty} \sum_{j=1}^{n} \alpha_{j}(t) k_{j}(t) d t<\infty
\end{array}\right.
$$

where $\delta$ is a fixed positive number. Moreover, define

$$
\mu_{1}(t)=L-\psi(t), \quad \mu_{2}(t)=L+\psi(t)
$$

where $L \in \mathbb{R}$ is a fixed number. By the properties of $\alpha_{i}$ and $k_{i}, i=1, \ldots, n$, the functions $\mu_{1}, \mu_{2}$ are well defined on $I, \psi>0$ on $I, \mu_{1}(t)<\mu_{2}(t)$ on $I$ and

$$
\lim _{t \rightarrow \infty} \mu_{j}(t)=L, \quad j=1,2 .
$$

We show that the set $\omega=\left\{(t, y) \in \omega_{0}: l_{1}(t, y)<0\right\}$ where $\omega_{0}=\left(t_{0}, \infty\right) \times \mathbb{R}$ and $l_{1}(t, y)=\left(y-\mu_{1}(t)\right)\left(y-\mu_{2}(t)\right)$ is a regular polyfacial set with respect to (1.1). Set $n=1$. Condition $(\alpha)$ of Definition 1 is obviously satisfied for $\Omega=\left(t_{0}, \infty\right) \times C$. For $(\beta)$, we have, for $t>t_{0}+r$,

$$
\begin{aligned}
D l_{1}(t, y)= & {\left[\sum_{i=1}^{n} \alpha_{i}(t)\left[\pi(0)-\pi\left(-\tau_{i}(t)\right)\right]-\mu_{1}^{\prime}(t)\right]\left[\pi(0)-\mu_{2}(t)\right] } \\
& +\left[\pi(0)-\mu_{1}(t)\right]\left[\sum_{i=1}^{n} \alpha_{i}(t)\left[\pi(0)-\pi\left(-\tau_{i}(t)\right)\right]-\mu_{2}^{\prime}(t)\right] .
\end{aligned}
$$

Since $\mu_{1}(t+\theta)<\pi(\theta)<\mu_{2}(t+\theta)$ for $\theta \in[-r, 0)$ and $\pi(0)=\mu_{1}(t)$ or $\pi(0)=\mu_{2}(t)$, we have, in view of (1.2) (if $\left.\pi(0)=\mu_{2}(t), t>t_{0}+r\right)$,

$$
\begin{aligned}
D l_{1}(t, y)> & \left(\mu_{2}(t)-\mu_{1}(t)\right)\left[\sum_{i=1}^{n} \alpha_{i}(t)\left[\mu_{2}(t)-\mu_{2}\left(t-\tau_{i}(t)\right)\right]-\mu_{2}^{\prime}(t)\right] \\
= & 2 \psi(t)\left[\sum_{i=1}^{n} \alpha_{i}(t)\left[L+\psi(t)-L-\psi\left(t-\tau_{i}(t)\right)\right]-\psi^{\prime}(t)\right] \\
= & 2 \delta \psi(t) \exp \left[-\int_{t_{0}}^{t} \sum_{j=1}^{n} \alpha_{j}(s) k_{j}(s) d s\right] \\
& \times \sum_{i=1}^{n} \alpha_{i}(t)\left[1+k_{i}(t)-\exp \left[\int_{t-\tau_{i}(t)}^{t} \sum_{j=1}^{n} \alpha_{j}(s) k_{j}(s) d s\right]\right] \geq 0
\end{aligned}
$$

and, consequently, $D l_{1}(t, y)>0$ for $t>t_{0}+r$. By analogy we get $D l_{1}(t, y)>$ 
0 if $\pi(0)=\mu_{1}(t)$ and $t>t_{0}+r$. So $(\beta)$ holds. In our case $q=0$ and condition $(\gamma)$ is not considered. Thus all conditions of Definition 1 are satisfied.

Now we verify the assumptions of Lemma 3. In our case

$W=\left\{(t, y) \in \partial \omega: m_{j}(t, y)<0, j=1, \ldots, q\right\} \equiv\left\{(t, y) \in \partial \omega: l_{1}(t, y)=0\right\}$.

Put $Z=\left\{(t, y) \in \bar{\omega}: t=t_{0}+2 r\right\}$. The previous computations were independent of the choice of the positive number $\delta$. Take now

$$
\delta>M\left\{\min _{\left[t_{0}+r, t_{0}+2 r\right]}\left[\exp \left[-\int_{t_{0}}^{t} \sum_{j=1}^{n} \alpha_{j}(s) k_{j}(s) d s\right] \sum_{j=1}^{n} \alpha_{j}(t) k_{j}(t)\right]\right\}^{-1}
$$

with $M=\max _{\left[t_{0}+r, t_{0}+2 r\right]}\left|\left(y\left(t_{0}, \varphi\right)(t)\right)^{\prime}\right|$. In this case, as can be shown easily,

$$
\left|\left(y\left(t_{0}, \varphi\right)(t)\right)^{\prime}\right|<\left|\psi^{\prime}(t)\right|
$$

for each $t \in\left[t_{0}+r, t_{0}+2 r\right]$. Thus we can define a system of initial functions $p_{Z, \omega}$ which satisfies the conditions of Definition 2 by

$$
p(z)=p(t, \varepsilon)=y\left(t_{0}, \varphi\right)(t)+\varepsilon
$$

for all $t \in\left[t_{0}+r, t_{0}+2 r\right]$ and some $\varepsilon \in\left[\mu_{1}\left(t_{0}+2 r\right)-y\left(t_{0}, \varphi\right)\left(t_{0}+2 r\right)\right.$, $\left.\mu_{2}\left(t_{0}+2 r\right)-y\left(t_{0}, \varphi\right)\left(t_{0}+2 r\right)\right]$. Define $\Pi: W \rightarrow Z \cap W$ by

$$
\Pi: W \ni\left(t^{*}, y^{*}\right) \mapsto\left(t_{0}+2 r, y^{* *}\right) \in Z \cap W
$$

where

$$
y^{* *}=\mu_{2}\left(t_{0}+2 r\right) \operatorname{sign}\left(y^{*}-\mu_{1}\left(t^{*}\right)\right)+\mu_{1}\left(t_{0}+2 r\right) \operatorname{sign}\left(\mu_{2}\left(t^{*}\right)-y^{*}\right) .
$$

Clearly, $\Pi$ is continuous and is the identity on $Z \cap W$, so $Z \cap W$ is a retract of $W$. Finally, $Z \cap W$ is not a retract of $Z$ because the boundary of an $n$ dimensional disc is not a retract of the disc (see e.g. [4]). By Lemma 3 there exists $z_{0}=\left(t_{0}+2 r, y_{0}\right) \in Z \cap \omega$ such that the graph of the corresponding solution $y=\xi(t)$ of (1.1) with initial condition $\left(t_{0}+2 r, p\left(z_{0}\right)\right)$ lies in $\omega$ for all $t \in D_{t_{0}+2 r, p\left(z_{0}\right)} \equiv \widetilde{I}_{1}=\left[t_{0}+2 r, \infty\right)$. That is, $\mu_{1}(t)<\xi(t)<\mu_{2}(t)$ on $\widetilde{I}_{1}$ or $|\xi(t)-L|<\psi(t)$ on $\widetilde{I}_{1}$. Since $\psi(\infty)=0$, we have $\lim _{t \rightarrow \infty} \xi(t)=L$. Clearly $\xi(t) \equiv p(t, \varepsilon)$ for some $\varepsilon=\varepsilon^{*} \in\left[\mu_{1}\left(t_{0}+2 r\right)-y\left(t_{0}, \varphi\right)\left(t_{0}+2 r\right), \mu_{2}\left(t_{0}+2 r\right)-\right.$ $\left.y\left(t_{0}, \varphi\right)\left(t_{0}+2 r\right)\right]$. Therefore, by $(4.2)$,

$$
y\left(t_{0}, \varphi\right)(t)=\xi(t)-\varepsilon^{*}
$$

for all $t \geq t_{-1}$ and, consequently,

$$
\lim _{t \rightarrow \infty} y\left(t_{0}, \varphi\right)(t)=L-\varepsilon^{*} .
$$

This means that each solution converges.

Proof of Theorem 2. This proof can be done in a similar fashion to the proof of the necessity of Theorem 1 if we take into consideration that the functions $k_{i}(t), i=1, \ldots, n$, defined by (4.1) satisfy $(1.3)$ and $k_{i}(\infty)=0$. 
Proof of Theorem 3. The assertion follows from the proof of Theorem 1. Indeed, the existence of a nonconstant monotone convergent solution of (1.1) corresponding to the initial point $t_{0}$ yields (in view of Lemma 1 ) the existence of a convergent, decreasing on $I_{-1}$, solution $y=y(t)$ of (1.1) with positive limit, corresponding to the initial point $t_{0}$. Consequently, this solution satisfies the conditions required in the necessity part of the proof of Theorem 1. Then the positive functions $k_{i}(t), i=1, \ldots, n, t \in I$, given by (4.1), satisfying (1.2), exist. The remaining part of the proof follows from Theorem 1.

Proof of Theorem 4. Necessity. Assume there is a solution of (1.1) defined on $I_{-1}$ with $y(\infty)=\infty$. Then there is no solution of (1.2) on $I_{1}$ with the above indicated properties since in the opposite case we get a contradiction with Theorem 1.

Sufficiency. Suppose that there is no solution of (1.2) with the indicated properties. Then, by Lemma 2 , the solution $y\left(t_{0}, \varphi\right)(t)$ generated by the initial function $\varphi(t)$ on $\left[t_{-1}, t_{0}\right]$ which satisfies condition (3.1) for $p=-1$ is increasing on $I$. If the limit of this solution is finite, then, by the proof of necessity of Theorem 1, there is a solution of (1.2) with the indicated properties (in this part of the proof we use the solution $w(t) \equiv-y(t)$, shifted by a constant if necessary, instead of the solution $y(t))$. This is a contradiction with Theorem 1.

Proof of Theorem 5. Necessity. Suppose each solution of (1.1) is convergent. Then by Theorem 2 there is a solution of (1.3) and we get

$$
1+k_{i}(t) \geq \exp \left[b \int_{t-\widetilde{r}}^{t} \sum_{j=1}^{n} k_{j}(s) d s\right], \quad i=1, \ldots, n, t \in I_{1} .
$$

Set $k(t)=\min _{i}\left\{k_{i}(t)\right\}$. Since

$$
\lim _{t \rightarrow \infty} k(t)=\lim _{t \rightarrow \infty} k_{i}(t)=0, \quad i=1, \ldots, n,
$$

and $k(t)>0$ on $I$, there is $t^{*} \in I_{1}$ such that $k\left(t^{*}-\theta \tau\left(t^{*}\right)\right) \geq k\left(t^{*}\right)$ for all $\theta \in[0,1]$. Moreover, there is $i \in\{1, \ldots, n\}$ such that $k_{i}\left(t^{*}\right)=k\left(t^{*}\right)$. Therefore

$$
1+k\left(t^{*}\right) \geq e^{n b \widetilde{r} k\left(t^{*}\right)} .
$$

This is only possible if $n b \widetilde{r}<1$ and so (1.4) holds.

Sufficiency. We use Theorem 1. We verify that the inequalities (1.2) for $k_{i}(t) \equiv k, i=1, \ldots, n$, hold where $k$ is a positive solution of the equation $1+k=e^{n a r k}$ (its existence follows from (1.5)). Indeed, obviously

$$
1+k=e^{n a r k} \geq \exp \left[\int_{t-\tau_{i}(t)}^{t} \sum_{j=1}^{n} \alpha_{j}(s) k d s\right], \quad i=1, \ldots, n, t \in I_{1} .
$$


Proof of Theorem 6. This theorem follows immediately from Theorem 1 if $k_{i}(t) \equiv k, i=1, \ldots, n$, where $k$ is a sufficiently small positive number and $t_{0}$ is sufficiently large. Indeed, in this case we can (without loss of generality) suppose that on $I_{1}$,

$$
\sum_{j=1}^{n} \int_{t-\tau_{i}(t)}^{t} \alpha_{j}(s) d s \leq q<1
$$

for all $i=1, \ldots, n$ and some $q \in\left(\max _{i}\left\{\sum_{j=1}^{n} q_{j i}\right\}, 1\right)$. Then there is $k \in \mathbb{R}^{+}$ such that

$$
1+k \geq e^{k q} \geq \exp \left[k \int_{t-\tau_{i}(t)}^{t} \sum_{j=1}^{n} \alpha_{j}(s) d s\right], \quad i=1, \ldots, n, t \in I_{1} .
$$

Proof of Theorem \%. In view of (2.1) the inequality

$$
\sum_{j=1}^{n} \int_{t-\tau_{i}(t)}^{t} \alpha_{j}(s) d s \geq q \geq 1
$$

holds on $I_{1}$ for all $i=1, \ldots, n$ and some $q \in\left[1, \min _{i}\left\{\sum_{j=1}^{n} q_{i j}\right\}\right]$. Suppose that there is no solution of (1.1) with $y(\infty)=\infty$. Then by Theorem 4 there is a positive solution of (1.2). Therefore, by Theorem 1, all solutions of (1.1) are convergent and by Theorem 2 there are functions $k_{i} \in C\left(I, \mathbb{R}^{+}\right)$, $i=1, \ldots, n$, which satisfy (1.3). Set $k(t)=\min _{i}\left\{k_{i}(t)\right\}$. As in the proof of necessity of Theorem 5 , there is $t^{*} \in I_{1}$ such that $k\left(t^{*}-\theta \tau\left(t^{*}\right)\right) \geq k\left(t^{*}\right)$ for all $\theta \in[0,1]$. Moreover, there is $i \in\{1, \ldots, n\}$ such that $k_{i}\left(t^{*}\right)=k\left(t^{*}\right)$. From (1.3) we get

$$
\begin{aligned}
1+k_{i}\left(t^{*}\right) & =\exp \left[\int_{t^{*}-\tau_{i}\left(t^{*}\right)}^{t^{*}} \sum_{j=1}^{n} \alpha_{j}(s) k_{j}(s) d s\right] \\
& \geq \exp \left[k\left(t^{*}\right) \int_{t^{*}-\tau_{i}\left(t^{*}\right)}^{t^{*}} \sum_{j=1}^{n} \alpha_{j}(s) d s\right] \geq e^{q k\left(t^{*}\right)}, \quad i=1, \ldots, n .
\end{aligned}
$$

We obtain a contradiction since $1+k\left(t^{*}\right) \geq e^{q k\left(t^{*}\right)}$ with $k\left(t^{*}\right)>0$ is impossible.

Proof of Theorem 8. Without loss of generality, suppose $\tau_{i} \leq r, j=$ $1, \ldots, n, t>r$ and $t$ sufficiently large. Set

$$
k_{j}(t)=\frac{\varepsilon}{t}\left(\frac{1}{\tau_{j}}-\frac{L_{j}}{t}\right)^{-1}, \quad j=1, \ldots, n,
$$

where $\varepsilon>0$. Let us verify inequalities (1.2). The $i$ th left hand side of (1.2) 
has the asymptotic expansion

$$
\mathcal{L}_{i}(t)=1+k_{i}(t)=1+\frac{\varepsilon \tau_{i}}{t\left(1-L_{i} \tau_{i} / t\right)}=1+\frac{\varepsilon \tau_{i}}{t}+\frac{\varepsilon L_{i} \tau_{i}^{2}}{t^{2}} \cdot(1+o(1)) .
$$

Now we estimate the $i$ th right hand side $\mathcal{R}_{i}(t)$ of (1.2). We have

$$
\begin{aligned}
\mathcal{R}_{i}(t) & =\exp \left[\int_{t-\tau_{i}(t)}^{t} \sum_{j=1}^{n} \alpha_{j}(s) k_{j}(s) d s\right] \leq \exp \left[\varepsilon \int_{t-\tau_{i}}^{t} \frac{1}{s} d s\right] \\
& =\exp \left[\varepsilon \ln \frac{t}{t-\tau_{i}}\right]=\left(1-\frac{\tau_{i}}{t}\right)^{-\varepsilon} \\
& =1+\frac{\varepsilon \tau_{i}}{t}+\frac{\varepsilon+1}{2} \cdot \varepsilon \cdot \frac{\tau_{i}^{2}}{t^{2}} \cdot(1+o(1)) .
\end{aligned}
$$

We conclude that (1.2) will hold (supposing $t_{0}$ is sufficiently large) if

$$
\begin{aligned}
\mathcal{L}_{i}(t) & =1+\frac{\varepsilon \tau_{i}}{t}+\frac{\varepsilon L_{i} \tau_{i}^{2}}{t^{2}} \cdot(1+o(1)) \\
& >1+\frac{\varepsilon \tau_{i}}{t}+\frac{\varepsilon+1}{2} \cdot \varepsilon \cdot \frac{\tau_{i}^{2}}{t^{2}} \cdot(1+o(1)) \geq \mathcal{R}_{i}(t), \quad i=1, \ldots, n .
\end{aligned}
$$

Comparing corresponding terms, we can see that this will hold when $L_{i}>$ $(\varepsilon+1) / 2$. Since $\varepsilon>0$ may be chosen arbitrarily small, we get the condition $L_{i}>1 / 2$.

Proof of Theorem 9. Without loss of generality, suppose $\tau \leq r, t>$ $\max \{1, \tau\}$ and $t$ is sufficiently large. Set $k_{j}(t) \equiv k(t), j=1, \ldots, n$, where

$$
k(t)=\frac{1}{t \ln t\left(\ln _{2} t\right)^{\varepsilon}}
$$

and $\varepsilon>1$ is a constant. Obviously, (1.2) will hold if

$$
\mathcal{L}(t) \equiv 1+k(t) \geq \mathcal{R}(t) \equiv \exp \left[\int_{t-\tau}^{t} \sum_{j=1}^{n} \alpha_{j}(s) k(s) d s\right] .
$$

We estimate the right hand side $\mathcal{R}(t)$ of (4.3). By (2.4), we have

$$
\mathcal{R}(t) \leq \exp \left(\mathcal{R}^{\star}(t)\right)
$$

where

$$
\begin{aligned}
\mathcal{R}^{\star}(t) \equiv & \frac{1}{(t-\tau) \ln (t-\tau)\left(\ln _{2}(t-\tau)\right)^{\varepsilon}} \\
& \times\left(1-\frac{\tau}{t-\tau}-\frac{\tau}{(t-\tau) \ln (t-\tau)}-\frac{L}{(t-\tau)(\ln (t-\tau))\left(\ln _{2}(t-\tau)\right)}\right) .
\end{aligned}
$$


Let us find the asymptotic expansion of $\mathcal{R}^{\star}(t)$. First, we clearly have

$$
\begin{aligned}
\frac{1}{t-\tau} & =\frac{1}{t}\left(1+\frac{\tau}{t}+\frac{\tau^{2}}{t^{2}}+o\left(\frac{1}{t^{2}}\right)\right) \\
\frac{1}{\ln (t-\tau)} & =\frac{1}{\ln t}\left(1+\frac{\tau}{t \ln t}+\frac{\tau^{2}}{2 t^{2} \ln t}+o\left(\frac{1}{t^{2} \ln t}\right)\right) \\
\frac{1}{\ln _{2}(t-\tau)} & =\frac{1}{\ln _{2} t}\left(1+\frac{\tau}{t \ln t \ln _{2} t}+\frac{\tau^{2}}{2 t^{2} \ln t \ln _{2} t}+o\left(\frac{1}{t^{2} \ln t \ln _{2} t}\right)\right) .
\end{aligned}
$$

Thus

$$
\begin{aligned}
\mathcal{R}^{\star}(t)= & \frac{1}{t \ln t\left(\ln _{2} t\right)^{\varepsilon}}\left(1+\frac{\tau}{t}+\frac{\tau^{2}}{t^{2}}+o\left(\frac{1}{t^{2}}\right)\right) \\
& \times\left(1+\frac{\tau}{t \ln t}+\frac{\tau^{2}}{2 t^{2} \ln t}+o\left(\frac{1}{t^{2} \ln t}\right)\right) \\
& \times\left(1+\frac{\varepsilon \tau}{t \ln t \ln _{2} t}+\frac{\varepsilon \tau^{2}}{2 t^{2} \ln t \ln _{2} t}+o\left(\frac{1}{t^{2} \ln t \ln _{2} t}\right)\right) \\
& \times\left(1-\frac{\tau}{t}-\frac{\tau^{2}}{t^{2}}+o\left(\frac{1}{t^{2}}\right)-\frac{\tau}{t \ln t}\left(1+\frac{\tau}{t}+o\left(\frac{1}{t}\right)\right)\right. \\
& \times\left(1+\frac{\tau}{t \ln t}+o\left(\frac{1}{t \ln t}\right)\right) \\
& -\frac{L}{t \ln t \ln 2 t}\left(1+\frac{\tau}{t}+o\left(\frac{1}{t}\right)\right)\left(1+\frac{\tau}{t \ln t}+o\left(\frac{1}{t \ln t}\right)\right) \\
& \left.\times\left(1+\frac{\tau}{t \ln t \ln _{2} t}+o\left(\frac{1}{t \ln t \ln _{2} t}\right)\right)\right) \\
= & \frac{1}{t \ln t\left(\ln _{2} t\right)^{\varepsilon}}\left(1+\frac{\varepsilon \tau-L}{t \ln t \ln _{2} t}-\frac{\tau^{2}}{t^{2}}+o\left(\frac{1}{t^{2}}\right)\right) .
\end{aligned}
$$

Finally, we get

$$
\begin{aligned}
\exp \left(\mathcal{R}^{\star}(t)\right)= & 1+\frac{1}{t \ln t\left(\ln _{2} t\right)^{\varepsilon}}\left(1+\frac{\varepsilon \tau-L}{t \ln t \ln _{2} t}-\frac{\tau^{2}}{t^{2}}+o\left(\frac{1}{t^{2}}\right)\right) \\
& +\frac{1}{2 t^{2} \ln ^{2} t\left(\ln _{2} t\right)^{2 \varepsilon}}\left(1+\frac{\varepsilon \tau-L}{t \ln t \ln _{2} t}-\frac{\tau^{2}}{t^{2}}+o\left(\frac{1}{t^{2}}\right)\right)^{2}+\ldots \\
= & 1+\frac{1}{t \ln t\left(\ln _{2} t\right)^{\varepsilon}}+\frac{\varepsilon \tau-L}{t^{2} \ln ^{2} t \ln _{2}^{1+\varepsilon} t}+\frac{1}{2 t^{2} \ln ^{2} t \ln _{2}^{2 \varepsilon} t}+o\left(\frac{1}{t^{3}}\right) .
\end{aligned}
$$

For the validity of (4.3) it is sufficient to suppose that $\mathcal{L}(t) \geq \exp \left(\mathcal{R}^{\star}(t)\right)$ (for sufficiently large $t$ ), i.e. that

$$
1+\frac{1}{t \ln t\left(\ln _{2} t\right)^{\varepsilon}} \geq 1+\frac{1}{t \ln t\left(\ln _{2} t\right)^{\varepsilon}}+\frac{\varepsilon \tau-L}{t^{2} \ln ^{2} t \ln _{2}^{1+\varepsilon} t}+\frac{1}{2 t^{2} \ln ^{2} t \ln _{2}^{2 \varepsilon} t}+o\left(\frac{1}{t^{3}}\right) \text {. }
$$


This will hold (we take into account the supposition $\varepsilon>1$ ) if $L>\varepsilon \tau$. Since $\varepsilon$ may be chosen arbitrarily close to 1 , we obtain the condition $L>\tau$.

Proof of Theorem 10. Without loss of generality, suppose $\tau \leq r, t>\tau$ and $t$ is sufficiently large. Set $k_{j}(t) \equiv k(t), j=1, \ldots, n$, where

$$
k(t)=1 / t^{m+p}
$$

and $p$ is a positive constant. Obviously, (1.2) will be satisfied if, as above, the inequality (4.3) holds. Let us find the asymptotic expansion of the right hand side of (4.3) as $t \rightarrow \infty$. We get

$$
\begin{aligned}
\mathcal{R}(t)= & \exp \left[\int_{t-\tau}^{t}\left(\sum_{j=1}^{n} \alpha_{j}(s)\right) \frac{1}{s^{m+p}} d s\right] \\
\leq & \exp \left[\frac{1}{(t-\tau)^{m+p}}-\frac{L}{(t-\tau)^{m+p+1}}\right] \\
= & 1+\frac{1}{(t-\tau)^{m+p}}-\frac{L}{(t-\tau)^{m+p+1}}+\frac{1}{2(t-\tau)^{2(m+p)}}+\ldots \\
= & 1+\frac{1}{t^{m+p}}\left(1-\frac{\tau}{t}\right)^{-(m+p)} \\
& -\frac{L}{t^{m+p+1}}\left(1-\frac{\tau}{t}\right)^{-(m+p+1)}+O\left(\frac{1}{t^{2(m+p)}}\right) \\
= & 1+\frac{1}{t^{m+p}}\left(1+\frac{(m+p) \tau}{t}+O\left(\frac{1}{t^{2}}\right)\right) \\
& -\frac{L}{t^{m+p+1}}\left(1+O\left(\frac{1}{t}\right)\right)+O\left(\frac{1}{t^{2(m+p)}}\right) \\
= & 1+\frac{1}{t^{m+p}}+\frac{(m+p) \tau-L}{t^{m+p+1}}+O\left(\frac{1}{t^{m+p+2}}\right)+O\left(\frac{1}{t^{2(m+p)}}\right) .
\end{aligned}
$$

Now, for

$$
\begin{aligned}
\mathcal{L}(t) & \equiv 1+\frac{1}{t^{m+p}} \\
& \geq 1+\frac{1}{t^{m+p}}+\frac{(m+p) \tau-L}{t^{m+p+1}}+O\left(\frac{1}{t^{m+p+2}}\right)+O\left(\frac{1}{t^{2(m+p)}}\right) \geq \mathcal{R}(t)
\end{aligned}
$$

to hold, $m+p>1$ and $L>(m+p) \tau$ are sufficient. Since $p$ may be chosen arbitrarily small positive, we obtain the condition $L>\tau m$.

Acknowledgements. This work was supported by grants 201/96/0410 and 201/99/0295 of Czech Grant Agency (Prague). The author is grateful to the referee for his suggestions to improve the presentation of this paper. 


\section{References}

[1] O. Arino, I. Györi and M. Pituk, Asymptotically diagonal delay differential systems, J. Math. Anal. Appl. (in the press).

[2] F. V. Atkinson and J. R. Had dock, Criteria for asymptotic constancy of solutions of functional differential equations, J. Math. Anal. Appl. 91 (1983), 410-423.

[3] R. Bellman and K. L. Cooke, Differential-Difference Equations, Academic Press, New York, 1963.

[4] K. Borsuk, Theory of Retracts, PWN, Warszawa, 1967.

[5] J. Čermák, On the asymptotic behaviour of solutions of certain functional differential equations, Math. Slovaca 48 (1998), 187-212.

[6] - The asymptotic bounds of solutions of linear delay systems, J. Math. Anal. Appl. 225 (1998), 373-388.

[7] J. Diblík, Asymptotic representation of solutions of equation $\dot{y}(t)=\beta(t)[y(t)-$ $y(t-\tau(t))]$, ibid. 217 (1998), 200-215.

[8] I. Győri and M. Pituk, Comparison theorems and asymptotic equilibrium for delay differential and difference equations, Dynam. Systems Appl. 5 (1996), 277-302.

[9] - - - $L^{2}$-perturbation of a linear delay differential equation, J. Math. Anal. Appl. 195 (1995), 415-427.

[10] J. K. Hale and S. M. V. Lunel, Introduction to Functional Differential Equations, Springer, 1993

[11] T. Krisztin, Asymptotic estimation for functional differential equations via Lyapunov functions, J. Math. Anal. Appl. 109 (1985), 509-521.

[12] - On the rate of convergence of solutions of functional differential equations, Funkcial. Ekvac. 29 (1986), 1-10.

[13] - , A note on the convergence of the solutions of a linear functional differential equation, J. Math. Anal. Appl. 145 (1990), 17-25.

[14] F. Neuman, On equivalence of linear functional-differential equations, Results in Math. 26 (1994), 354-359.

[15] -, On transformations of differential equations and systems with deviating argument, Czechoslovak Math. J. 31 (1981), 87-90.

[16] K. P. Rybakowski, Ważewski's principle for retarded functional differential equations, J. Differential Equations 36 (1980), 117-138.

[17] T. Ważewski, Sur un principe topologique de l'examen de l'allure asymptotique des intégrales des équations différentielles ordinaires, Ann. Soc. Polon. Math. 20 (1947), 279-313.

[18] S. N. Zhang, Asymptotic behaviour and structure of solutions for equation $\dot{x}(t)=$ $p(t)[x(t)-x(t-1)]$, J. Anhui Univ. (Natural Science Edition) 2 (1981), 11-21 (in Chinese).

Department of Mathematics

Faculty of Electrical Engineering and Computer Science

Technical University of Brno

Technická 8

61600 Brno, Czech Republic

E-mail:diblik@dmat.fee.vutbr.cz 